\title{
Review
}

\section{Molecular Basis for the Maintenance of Envelope Integrity in Selenomonas ruminantium: Cadaverine Biosynthesis and Covalent Modification into the Peptidoglycan Play a Major Role}

\author{
Seiji KoJIMA ${ }^{1, *}$ and Yoshiyuki KAMIO ${ }^{2, * *}$ \\ ${ }^{1}$ Laboratory of Applied Microbiology, Department of Microbial Biotechnology, Graduate School of Agricul- \\ tural Science, Tohoku University, Tsutsumi-dori Amamiya-machi 1-1, Aoba-ku, Sendai 981-8555, Japan \\ ${ }^{2}$ Department of Biochemical Engineering, Graduate School of Science and Engineering, Yamagata University, \\ Jyonan 4-3-16, Yonezawa 992-8510, Japan
}

(Received February 9, 2012)

\begin{abstract}
Summary Polyamine is a small organic polycation composed of a hydrocarbon backbone with multiple amino groups which ubiquitously exists in all living organisms from bacteria to higher animals. The critical step of polyamine biosynthesis generally includes the amino acid-decarboxylating reaction to produce the primary diamines, such as a synthesis of putrescine $\left(\mathrm{NH}_{3}{ }^{+} \cdot\left(\mathrm{CH}_{2}\right)_{4} \cdot \mathrm{NH}_{3}{ }^{+}\right)$from ornithine, and cadaverine $\left(\mathrm{NH}_{3}{ }^{+} \cdot\left(\mathrm{CH}_{2}\right)_{5} \cdot \mathrm{NH}_{3}{ }^{+}\right)$from lysine, which are catalyzed by pyridoxal-5'-phosphate (PLP; vitamin $\mathrm{B}_{6}$ )-dependent decarboxylases. Synthesized polyamines are implicated in a wide variety of cytoplasmic reactions such as DNA replication and protein synthesis, and are essential for proper growth of the organisms. However, in Selenomonas ruminantium, a strictly anaerobic Gram-negative bacterium dominant in sheep rumen, cadaverine displays its function in a quite distinctive scheme compared to the general bacteria reported. It serves as an essential constituent of the peptidoglycan for the maintenance of envelope integrity through an interaction with the periplasm-exposed SLH domain of Mep45, the outer membrane protein of this bacterium. Furthermore, cytoplasmic biosynthesis of cadaverine occurs totally in a eukaryotic-like manner rather than in a conventional way of bacteria. Lysine/ornithine decarboxylase (LDC/ODC), a PLP-dependent enzyme responsible for cadaverine synthesis in this bacterium, displays significant homology to the eukaryotic ODC but not to the general bacterial LDC nor ODC, and its activity is tightly regulated by antizyme-mediated proteolysis, a regulatory process generally found in eukaryotes. These findings represent the biological diversity of this bacterium beyond the preexisting knowledge related to the polyamine-physiology, cell envelope-architecture, and the regulatory system for the enzyme. In this review we will describe (i) the cadaverine-containing peptidoglycan of $S$. ruminantium: its chemical structure, biosynthesis, and biological function, and (ii) cellular biosynthesis of cadaverine by LDC/ODC and its antizyme-mediated regulation. In addition, we will briefly refer to (iii) the phylogenetic position and characteristics of $S$. ruminantium and its unique cadaverine-physiology.
\end{abstract}

Key Words Selenomonas ruminantium, cadaverine-containing peptidoglycan, cell envelope integrity, lysine decarboxylase, antizyme-mediated proteolysis

The cell envelope of bacteria represents the outermost boundary separating the interior of the cell from the surrounding environment (1). It protects the cell as a permeation barrier by limiting the influx of harmful molecules; meanwhile the substances essential for cell

\footnotetext{
* Present address: Department of Molecular and Cell Biology, Dr. H. Nikaido's Laboratory, University of California, Berkeley, 16 Barker Hall \# 3202, Berkeley, CA 94720-3202, USA

** To whom correspondence should be addressed at present address: Laboratory of Plant Molecular and Cellular Biology, Graduate School of Life Science, Tohoku University, 2-1-1 Katahira, Aoba-ku, Sendai 980-8577, Japan

E-mail: ykamio@ige.tohoku.ac.jp
}

proliferation are uptaken, or secreted, across the envelope via the transport machinery composed of a set of membrane protein(s). Furthermore, it provides the cell with sufficient solidity to maintain shape stability and tolerance against osmotic pressure. The envelope of Gram-negative bacteria is organized in three layers comprising cytoplasmic membrane, peptidoglycan layer, and outer membrane. To fulfill the envelope function properly, these layers must be constructed and translocated correctly, and structurally connected within each other to secure the stability and integrity of the cell surface (2-6). The structural connection between the outer membrane and the peptidoglycan has been investigated mainly in Escherichia coli, a model 
organism of the Gram-negative bacteria. It was shown that a murein-lipoprotein $(3,5,6)$ and multiproteinapparatus termed the Tol-Pal system $(2,4)$ are involved in the structural linkage between the outer membrane and the peptidoglycan. A mutant cell defective for either protein no longer maintains the cell surface integrity, displaying detachment of the outer membrane from the peptidoglycan and hypersensitivity to external damage agents. Since these proteins are widely distributed among the proteobacteria, a representative group of Gram-negative bacteria (7), these are regarded as the model systems for the structural connection.

However, Selenomonas ruminantium, a strictly anaerobic Gram-negative bacterium dominant in sheep rumen, is equipped with a completely different system to anchor the outer membrane to the peptidoglycan. In this bacterium, the murein-lipoprotein and Tol-Pal system are absent, but instead, cadaverine $\left(\mathrm{NH}_{3}{ }^{+} \cdot\left(\mathrm{CH}_{2}\right)_{5} \cdot \mathrm{NH}_{3}{ }^{+}\right)$, a kind of polyamine, is covalently bound to the peptidoglycan as an essential constituent and plays a significant role $(8-11)$. It mediates the interaction between the peptidoglycan and the periplasm-exposed S-layer homologous (SLH) domain of Mep45, a major outer membrane protein of this bacterium, thereby forming the structural linkage between the peptidoglycan and the outer membrane $(12,13)$. Polyamine is a small polycationic molecule composed of a hydrocarbon backbone with multiple amino groups. It exists ubiquitously in all living organisms, and participates in a wide variety of biological reactions such as DNA replication or protein synthesis (14). However, until the research by Kamio et al. (10) there had been no report regarding the cell wall-bound polyamine in bacteria.

In $S$. ruminantium, cadaverine biosynthesis takes place in the cytoplasm by decarboxylating ornithine into cadaverine catalyzed by lysine/ornithine decarboxylase (LDC/ODC), a pyridoxal-5'-phosphate (PLP)dependent enzyme (15-17). Cadaverine is subsequently transferred to the peptidoglycan at the intermediate stage of peptidoglycan-biosynthesis (18), and functions at the cell surface. During the research on the cellular biosynthesis and regulation of cadaverine, it was revealed that it works in a quite eukaryote-like manner, rather than in any conventional way of bacteria ever reported. LDC/ODC displays significant homology to the eukaryotic ODC but not to the general bacterial LDC nor ODC (17), and its activity is tightly regulated by antizyme-mediated proteolysis, a regulatory process generally found in eukaryotes (19-21). Taken together, in this bacterium cadaverine exerts its function in a quite distinctive scheme compared to the general bacteria, in respect of the unique biosynthesis, and utilization as a cell wall constituent.

Understanding the total physiology of cadaverine in S. ruminantium including biosynthesis, transferring reaction to the peptidoglycan, and physiological function at the cell surface, have been the fundamental research focus, and at present we have reached the status of knowledge necessary to describe it in precise outline. In this review we will describe (i) the cadaverine- containing peptidoglycan of $S$. ruminantium: its chemical structure, biosynthesis, and biological function, and (ii) cellular biosynthesis of cadaverine by LDC/ODC and its antizyme-mediated regulation. In addition, we will briefly refer to (iii) the phylogenetic position and characteristics of $S$. ruminantium and its unique "cadaverine-physiology."

\section{Cadaverine-Containing Peptidoglycan in S. rumi- nantium}

(i) Discovery of cadaverine covalently-linked to the peptidoglycan

At the beginning of the 1980s, the cell surface structure and envelope protein composition of S. ruminantium was investigated by Kamio and Takahashi (8). It was revealed that this bacterium possesses a typical Gram-negative cell surface, comprising the cytoplasmic membrane, peptidoglycan, and the outer membrane, but is characterized by the exceptional feature of the absence of murein-lipoprotein. Murein-lipoprotein plays an important role in the structural linkage between the outer membrane and peptidoglycan, thereby maintaining the structural integrity of the cell surface of Gram-negative bacteria $(3,5)$. The E. coli lpo mutant, which lacks murein-lipoprotein, becomes hypersensitive to EDTA, resulting in rapid cell lysis upon the exposure to it (5). However, the S. ruminantium cell was found to be resistant to EDTA despite the absence of murein-lipoprotein. This phenotypic disagreement led to the assumption that some substance(s) might play a similar role to the murein-lipoprotein, and further investigation of the peptidoglycan component was carried out. The $\mathrm{HCl}$-hydrolysate of the ${ }^{14}[\mathrm{C}]$-amino acidslabeled peptidoglycan of $S$. ruminantium was analyzed by cellulose thin-layer chromatography, and it was found that there was a radioactive spot besides Dglutamic acid, meso-diaminopimelic acid (meso-DAP), and DL-alanine, with a ninhydrin-positive property. The compound corresponding to this spot was isolated and analyzed, and was identified as cadaverine dihydrochloride $\left(\mathrm{NH}_{2}\left(\mathrm{CH}_{2}\right)_{5} \mathrm{NH}_{2} \cdot 2 \mathrm{HCl}\right)(10)$. The chemical composition of the peptidoglycan was determined to be L-Ala, D-Ala, D-Glu, meso-DAP, cadaverine, $N$-acetylmuramic acid and $N$-acetylglucosamine, in a molar ratio of $1.0: 1.0: 1.0: 1.1: 0.9: 1.0$, respectively. Except for cadaverine, the chemical composition of the peptidoglycan was identical to that of E. coli (22).

(ii) Chemical structure of the peptidoglycan of S. ruminantium

To determine the chemical structure of the peptidoglycan of $S$. ruminantium, ${ }^{14}[\mathrm{C}]$-cadaverine-labeled peptidoglycan was digested by the Streptomyces albus Genzymes, which contain glycosidase, N-acetylmuramylL-alanine amidase, and endopeptidase, and the degraded products were analyzed (9). ${ }^{14}[\mathrm{C}]$-cadaverine was recovered in a peptide composed of L-Ala, D-Ala, DGlu, meso-DAP, and cadaverine, in a molar ratio of $0.98: 1.0: 1.0: 0.98: 0.97$, respectively. The N-terminal residues of this peptide were meso-DAP, L-Ala, and cadaverine. Thus cadaverine was considered to bind to 


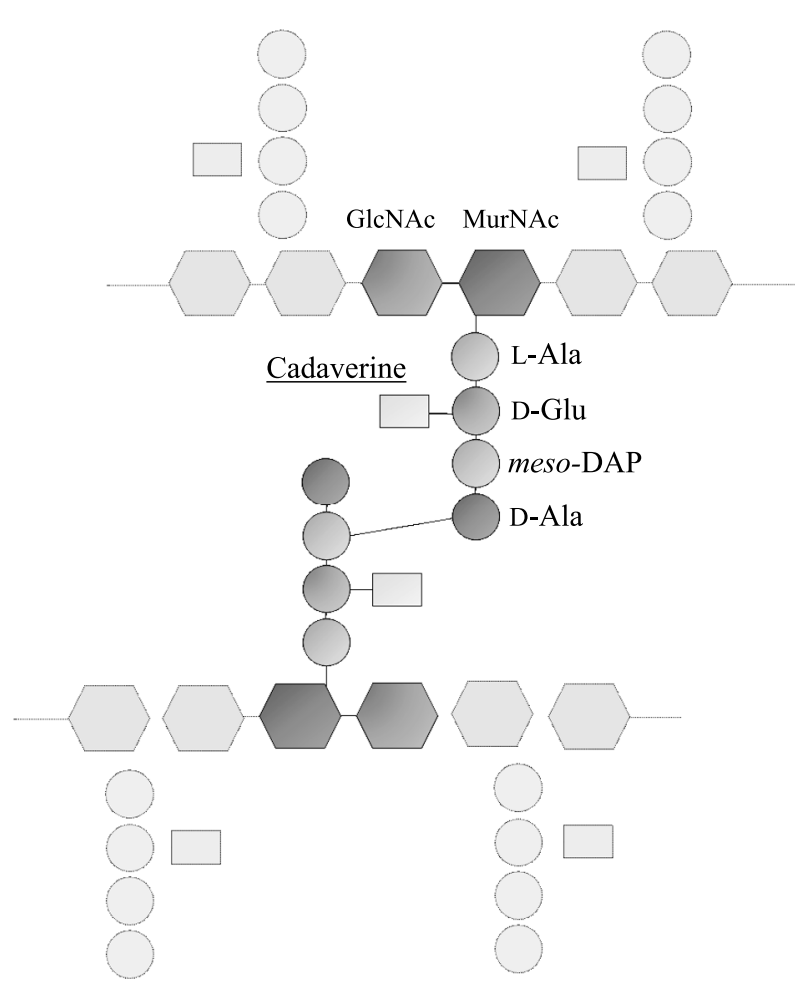

Fig. 1. Chemical structure of the cadaverine-containing peptidoglycan of $S$. ruminantium. Cadaverine binds to $\alpha$ carboxyl group of D-glutamic acid residue of the peptide stem.

this peptide by one of its two amino groups, the other remaining as a free cation. To identify the residue cadaverine links covalently, this peptide was subjected to partial acid hydrolysis, and two peptide fragments were obtained. One of them was a dipeptide of meso-DAP-DAla, and the other was composed of L-Ala, D-Glu, and cadaverine, and its $\mathrm{N}$-terminal residues were L-Ala and cadaverine. On the basis of these results, it was determined that cadaverine links to the $\alpha$-carboxyl group of the D-glutamic acid residue in the tetrapeptide of L-AlaD-Glu-meso-DAP-D-Ala. Accordingly, the entire structure of peptidoglycan of $S$. ruminantium was proposed as shown in Fig. 1.

(iii) Biosynthesis of cadaverine-containing peptidoglycan

The peptidoglycan layer is a giant macromolecularmeshwork constructed by the polysaccharide chain composed of alternating units of $\mathrm{N}$-acetylmuramic acid (MurNAc) and N-acetylglucosamine (GlcNAc), and the peptide stem cross-linking the polysaccharide chains. In the biosynthetic process, GlcNAc-MurNAc-peptide unit serves as the monomeric precursor firstly synthesized, and it will be polymerized in a later process to form the entire structure of the peptidoglycan. The detailed process is divided into three stages: (i) synthesis of UDPGlcNAc and UDP-MurNAc-peptide in the cytoplasm, (ii) transferring these compounds to lipid (isoprenoid alcohol)-phosphate that resides at the inner side of the cytoplasmic membrane, followed by the formation of GlcNAc-MurNAc-peptide-lipid (lipid intermediate), (iii) translocation of lipid intermediate into the outer side of the cytoplasmic membrane, and the polymerization by transglycosylation and transpeptidation to form the entire structure of the peptidoglycan $(23,24)$.

The biosynthetic pathway of cadaverine-containing peptidoglycan of $S$. ruminantium was investigated by Kamio et al. (18). Firstly it was found that D-cycloserine and vancomycin caused $S$. ruminantium cells to accumulate the UDP-MurNAc-peptide in the cytoplasm; however, it contained no cadaverine. Therefore, the cadaverine-adding reaction was supposed to occur in a later stage at least after the lipid intermediate is formed. To define the reaction, the peptidoglycan-biosynthesis was reconstituted in vitro using the envelope preparation as a particulate enzyme. It was demonstrated that the cadaverine-adding reaction occurs ATP-dependently in the presence of the lipid intermediate as an acceptor for cadaverine. Thus, the incorporation of cadaverine into the peptidoglycan was concluded to take place at the internal side of the cell, at the intermediate stage of the peptidoglycan-biosynthesis. The particulate enzyme responsible for the cadaverine-adding reaction was designated as lipid intermediate:diamine transferase. Structural specificity of diamines required for transferring to the lipid intermediate was examined, and revealed that diamines with the structure of $\mathrm{NH}_{2}\left(\mathrm{CH}_{2}\right)_{n} \mathrm{NH}_{2} n=3$ to 7 could serve as the substrate for lipid intermediate:diamine transferase (25).

(iv) Biological function of the peptidoglycan-bound cadaverine

The biological function of cadaverine covalentlylinked peptidoglycan was firstly clarified by Kamio et al. using a selective inhibitor to block the activity of lysine/ ornithine decarboxylase (LDC/ODC), an enzyme responsible for cadaverine synthesis in S. ruminantium (11). In this aim, DL- $\alpha$-difluoromethyllysine (DFML), a potent and irreversible inhibitor of lysine decarboxylase originally shown in Mycoplasma dispar (26), was used. It was shown that DFML practically inhibits the $S$. ruminantium LDC/ODC activity both in vivo and in vitro. In the presence of the inhibitor, $S$. ruminantium cells showed a significant decrease in the amount of cadaverine covalently-linked to the peptidoglycan, and led to growth inhibition accompanying a drastic morphological change and aberrant cell surface structure with the detachment of the outer membrane from the peptidoglycan. The inhibitory effect was completely released by adding the external free cadaverine, which was exclusively incorporated into the peptidoglycan. On the basis of these results, cadaverine was considered to play a significant role in maintaining the integrity of the cell surface.

(v) Cadaverine-mediated anchoring mechanism between the outer membrane and the peptidoglycan

The mechanism for the maintenance of the cell surface integrity and the precise role of the peptidoglycanlinked cadaverine was investigated by Kojima et al., on the basis of the assumption that cadaverine associates with the structural connection between the outer membrane and the peptidoglycan, thereby serving as the functional counterpart of murein-lipoprotein (12). The outer membrane component to interact with the pepti- 


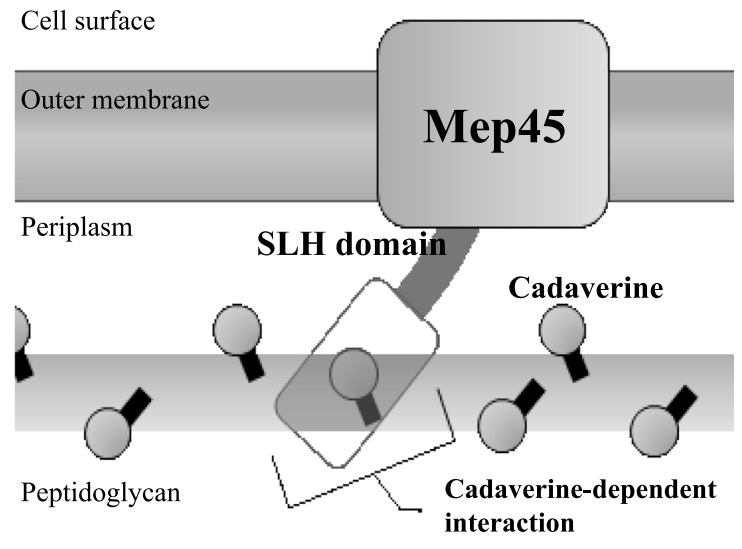

Inner membrane

Fig. 2. Schematic representation of the anchoring system between the outer membrane and the peptidoglycan in S. ruminantium. Peptidoglycan-linked cadaverine mediates the interaction between the periplasmexposed SLH domain of Mep45 and the peptidoglycan. This interaction serves as the structural linkage anchoring the outer membrane to the peptidoglycan.

doglycan-linked cadaverine was examined, and it was found that major outer membrane protein Mep45 acts as the binding-partner for the peptidoglycan in a manner dependent on the presence of peptidoglycan-linked cadaverine. Mep45 is a unique major outer membrane protein that is expected to cover the most part of the cell surface of this bacterium. It is comprised by the N-terminal S-layer homologous (SLH) domain exposed to the periplasmic space, and the C-terminal major region serving as the membrane-bound unit (Fig. 2). The SLH domain is the putative functional domain that interacts with various cell wall components and is widely distributed among the bacterial cell surface protein $(27,28)$. In vitro peptidoglycan-binding assay demonstrated that the N-terminal SLH domain is responsible for the interaction. It was shown that the SLH domain binds to the entire structure of the peptidoglycan including the cadaverine moiety, but cadaverine itself is not sufficient to serve as a binding ligand for the SLH domain. The high concentration of salt did not inhibit the binding; thus the interaction was considered to occur through the structure-based recognition for the cadaverine-containing peptidoglycan by the SLH domain, not by the ionic interaction.

The binding-strength between the SLH domain and the peptidoglycan in vitro was shown to be directly linked to the cell surface integrity in vivo, by using the series of the diamines with the structure of $\mathrm{NH}_{3}{ }^{+} \cdot\left(\mathrm{CH}_{2}\right)_{n} \cdot \mathrm{NH}_{3}{ }^{+} n=3$ to 6 (13). When S. ruminantium cell is cultivated in the condition inhibiting the cadaverine-biosynthesis, these diamines exogenously added to the culture were all capable of incorporating into the peptidoglycan at the same level. However, it was found that the binding affinity to the SLH domain was specifically high in the cadaverine $(n=5)$-containing peptidoglycan, compared to the peptidoglycan with other diamines. The cells containing peptidoglycanlinked diamines of $n=3,4$, or 6 showed lowered resistance to external damage agents in comparison to the cadaverine-containing cell, and displayed the detachment of the outer membrane from the peptidoglycan. On the basis of these findings, it was concluded that the functional significance of peptidoglycan-linked cadaverines is to facilitate the structural linkage anchoring the outer membrane to the peptidoglycan via the specific interaction with the SLH domain of Mep45, thereby maintaining the cell surface integrity in $S$. ruminantium (Fig. 2).

\section{Cadaverine Biosynthesis and Its Regulation in $S$. ruminantium}

\section{(i) Novel characteristics of lysine/ornithine decarboxylase (LDC/ODC) in S. ruminantium}

In $S$. ruminantium, cadaverine is synthesized by decarboxylating L-lysine. Kamio and Terawaki (15) purified PLP-dependent lysine decarboxylase from this bacterium and examined its basic properties. In most bacteria, lysine decarboxylase (LDC) functions mainly as an inducible enzyme that appears only under certain conditions such as low $\mathrm{pH}(29,30)$. However, LDC in $S$. ruminantium was suggested to be produced constitutively, based on the analyses of the enzyme activities in the cells grown under various conditions. Its molecular mass is $43 \mathrm{kDa}$ and it forms a homodimer of $88 \mathrm{kDa}$ as a native state. This is completely different from that of $E$. coli, whose LDC forms a $780 \mathrm{kDa}$ decameric structure consisting of identical subunits of $80 \mathrm{kDa}$.

In addition to these features, it was demonstrated that cadaverine synthesis in $S$. ruminantium can be inhibited not only by DFML but also by DL- $\alpha$-difluoromethylornithine (DFMO), an inhibitor specific for eukaryotic ornithine decarboxylase (31), implying the intriguing feature of the cadaverine-synthetic process in this bacterium (unpublished). The detailed enzymatic properties and the nucleotide sequence of the gene encoding $S$. ruminantium LDC was determined and investigated by Takatsuka et al. $(16,17)$. In vitro kinetic study using purified enzyme demonstrated that it shows characteristic substrate specificity with a capability for decarboxylating both L-lysine and L-ornithine, synthesizing cadaverine and putrescine. The $\mathrm{Km}$ for L-lysine and L-ornithine were $0.63 \mathrm{~mm}$ and $1.2 \mathrm{mM}$, respectively. The decarboxylating activity towards both substrates was competitively inhibited at the same time by either DFML or DFMO, providing a reasonable explanation for the unusual inhibitory effect of DFMO on in vivo cadaverine synthesis, and suggesting that the catalytic domain for both substrates is located in an identical region of this enzyme. According to these findings, the LDC of $S$. ruminantium has been designated as lysine/ornithine decarboxylase (LDC/ODC).

The nucleotide sequence of the gene encoding LDC/ ODC shows significant homology to eukaryotic ornithine decarboxylase (ODC) including that of mouse, 
Saccharomyces cerevisiae, Neurospora crassa, Trypanosoma brucei, and Caenorhabditis elegans, rather than general bacterial LDC (17). The amino acid residues implicated in the fundamental enzymatic function such as pyridoxal-5'-phosphate (PLP)-binding residues $\left(\mathrm{K}^{51}\right)$, substrate-binding residues $\left(\mathrm{C}^{323}\right.$ and $\left.\mathrm{D}^{324}\right)$, and residues responsible for dimer formation $\left(\mathrm{K}^{151}, \mathrm{D}^{116}, \mathrm{~K}^{275}, \mathrm{Y}^{290}\right.$, $\mathrm{Y}^{298}, \mathrm{D}^{327}, \mathrm{G}^{350}$ and $\mathrm{F}^{360}$ ), are all conserved in $\mathrm{LDC} /$ ODC. The PLP-dependent enzymes are generally classified into seven fold types based on the predicted secondary structure (32). LDC/ODC belongs to fold type III, which is the same type as the eukaryotic ODCs, contrary to the bacterial ODCs and LDCs, which belongs to fold type I. These findings indicate that LDC/ODC is totally similar to the eukaryotic ODCs rather than bacterial one, as judged from its nucleotide sequence. Since amino acid residues critical for the enzyme activity of eukaryotic ODCs are all conserved in LDC/ODC, the catalytic mechanism of this enzyme is considered to be similar to that of eukaryotic ODCs. Accordingly, $\mathrm{K}^{51}$ of LDC/ODC is supposed to be a PLP-binding residue forming an internal Schiff base between its $\varepsilon-\mathrm{NH}_{2}$ and the aldehyde group of PLP, and $\mathrm{C}^{323}$ to be the crucial residue for substrate binding, based on the evidence obtained from eukaryotic ODCs (33).

However, distinct from the eukaryotic ODCs which have exclusive substrate specificity towards L-ornithine, LDC/ODC displays dual specificity towards both L-lysine and L-ornithine. This functional diversity led to further investigation of the deduced active site of LDC/ODC. The regions neighboring $\mathrm{K}^{51}$ and $\mathrm{C}^{323}$ were examined and compared to the amino acid sequence of corresponding regions of mouse ODC (17). As a result, five amino acid residues $\left(A^{44}, G^{45}, V^{46}, P^{54}\right.$, and $\left.S^{322}\right)$ were identified to confer the dual-substrate specificity of this enzyme. It was demonstrated that replacing these five residues into the corresponding residues of mouse ODC (A44V/ G45T/V46P/P54D/S322A) converts the substrate specificity of LDC/ODC much preferable to L-ornithine (70 times higher than wild type LDC/ODC) (34). According to these findings, LDC/ODC was conceived as a sort of eukaryotic ODC, but containing the alteredcatalytic domain conferring the characteristic substrate specificity towards L-lysine, enabling the production of cadaverine (Fig. 3). This feature is quite distinctive from any bacterial LDCs or ODCs ever reported.

(ii) Antizyme-mediated regulation of $\mathrm{LDC} / \mathrm{ODC}$

During the research on LDC/ODC, it was discovered that LDC/ODC activity is drastically decreased at the early stationary phase, and this was revealed to occur due to the rapid proteolytic degradation of LDC/ODC (16). This degradation was ATP-dependent and inhibited by phenylmethylsulfonyl fluoride, strongly suggesting the existence of a proteolytic regulatory process for LDC/ODC. This phenomenon was quite reminiscent of antizyme-mediated regulation of ODCs in eukaryotes, in which the antizyme binds to monomeric ODC which is in a rapid equilibrium between the active dimeric form, and triggers the ATP-dependent proteolytic degradation by $26 \mathrm{~S}$ proteasome (35). In fact, LDC/ODC possesses the

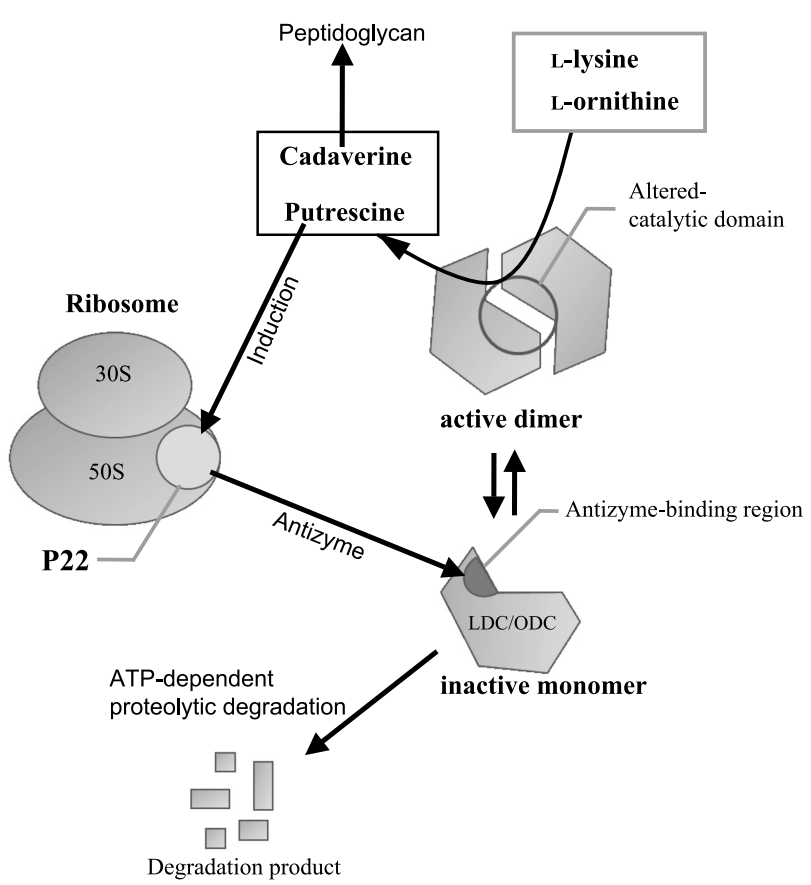

Fig. 3. Model of cadaverine biosynthesis and its antizyme-mediated regulation. Cadaverine is synthesized constitutively from L-lysine catalyzed by LDC/ODC. LDC/ODC displays eukaryotic ornithine decarboxylase (ODC)-like properties, but its catalytic domain is altered to confer the enzymatic activity towards both L-lysine and L-ornithine. The ribosomal protein L10 of this bacterium designated as $\mathrm{P} 22$ acts as an antizyme for LDC/ ODC. It binds to the antizyme-binding region of LDC/ ODC and triggers ATP-dependent proteolytic degradation. This antizyme-mediated regulatory system occurs at the early stationary phase, and also is inducible by the presence of putrescine.

antizyme-binding region of mouse ODC. These findings led to further investigation of the degradation mechanism of LDC/ODC.

Yamaguchi et al. isolated a $22 \mathrm{kDa}$ protein $(\mathrm{P} 22)$ as a stimulating factor for ATP-dependent proteolysis of LDC/ODC, and demonstrated that P22 acts completely as a counterpart of the eukaryotic antizyme $(19,20)$. It displays no degradation activity towards LDC/ODC, but binds to the antizyme-binding region of LDC/ODC and triggers ATP-dependent proteolysis (Fig. 3). This proteolytic system was totally compatible with the factors implicated in the antizyme-mediated regulatory system of mouse ODC, such as mouse antizyme and $26 \mathrm{~S}$ proteasome. Mouse antizyme can bind to the antizymebinding region of LDC/ODC, and triggers proteolysis. The $26 \mathrm{~S}$ proteasome can replace the degradation activity towards LDC/ODC whether triggered by P22 or by mouse antizyme. The $K_{D}(M)$ between P22 or mouse antizyme and LDC/ODC were $8.55 \times 10^{-11}$, and $10.8 \times 10^{-11}$, respectively. These findings indicate that LDC/ODC activity is regulated in a quite eukaryote-like manner. However, the nucleotide sequence of the gene encoding P22 was entirely different from that of mouse antizyme (20). It indicates that P22 is a ribosomal protein L10 of this bacterium, and indeed P22 was 
detected as the constituent of the 50S subunit of the ribosome. The L10 protein of E. coli has no antizyme activity like P22, and it was determined that two segments specifically exist in P22 $\left(\mathrm{K}^{101} \mathrm{NKLD}^{105}\right.$ and $\mathrm{G}^{160}$ VIRNAVYVLD ${ }^{170}$ ) are responsible for binding to $\mathrm{LDC} / \mathrm{ODC}$ (21). There is no report regarding such a regulatory system involving ribosome to serve as the bacterial antizyme. However, it remains unclear whether P22 can act as antizyme immediately as a state of ribosomal unit assembled inside the mature ribosome, or needs to be dissociated from ribosome to fulfill the antizyme function. Based on the genome-sequence analysis of $S$. ruminantium, there is no other putative L10 protein besides P22 (unpublished); thus it is expected that P22 plays duplicate roles both as the ribosomal protein and as the antizyme. The molecular mechanism for switching these duplicated functions of P22 needs to be elucidated in further research.

It appears noteworthy that this regulatory system is inducible by the presence of putrescine $\left(\mathrm{NH}_{3}{ }^{+} \cdot\left(\mathrm{CH}_{2}\right)_{4} \cdot \mathrm{NH}_{3}{ }^{+}\right)$. In $S$. ruminantium, putrescine is capable of being the substrate for lipid intermediate:diamine transferase and incorporated into the peptidoglycan; however, it is insufficient for the proper maintenance of the cell surface structure (13). Thus, one biological significance of this regulatory system is assumed to be the prevention of excessive putrescine synthesis by LDC/ODC.

\section{Phylogenetic Position of S. Ruminantium and Its Cadaverine-Related Physiology}

(i) Phylogenetic distribution of polyamine-containing peptidoglycan and the cell surface anchoring system

$S$. ruminantium is grouped into the Veillonellaceae family in the Clostridiales order of a firmicutes phylum by the $16 \mathrm{~S}$ rRNA-based classification. Firmicutes is constituted mostly by Gram-positive bacteria, but the bacteria in Veillonellaceae exceptionally possess Gram-negative cell surface structures. The peptidoglycan of the Veillonellaceae-belonging bacteria was examined and revealed that their peptidoglycan contains covalentlylinked polyamines (36-39). Putrescine and cadaverine are linked to the $V$. alcalescens, and $V$. parvula, and spermidine $\left(\mathrm{NH}_{3}{ }^{+} \cdot\left(\mathrm{CH}_{2}\right)_{3} \mathrm{NH}\left(\mathrm{CH}_{2}\right)_{4} \cdot \mathrm{NH}_{3}{ }^{+}\right)$are also found in Megasphaera elsdenii and Anaerovibrio lipolytica in addition to putrescine and cadaverine. Among them, $V$. alcalescens was particularly well investigated, and it was revealed that peptidoglycan-linked polyamines serve as the essential constituent for maintaining the cell surface integrity in this bacterium (36). The polyaminedepleted cell shows a phenotypic defect similar to the case in $S$. ruminantium cells, with a drastic morphological change and aberrant cell surface structure. Webbased homology search shows that a Mep45-like protein is conserved in this bacterium; thus the cell surface integrity is expected to be maintained in a manner similar to $S$. ruminantium, possibly through the specific interaction between the Mep45-like outer membrane protein and polyamine-containing peptidoglycan. In addition, the Mep45-like proteins are well distributed among Veillonellaceae, suggesting that the polyaminemediating anchoring system between the outer membrane and peptidoglycan is a general feature of this family.

In addition to the phylogenetic distribution of the anchoring mechanism in Veillonellaceae, the SLH domain of Mep45 shows significant homology to the corresponding domain of thremophilic bacteria such as Thermus thermophilus, and Thermotoga maritima, which have been regarded as ancient bacteria and are placed on the deepest branch of the phylogenetic tree (12). As long judged by $16 \mathrm{~S}$ rRNA-based classification, it seems that there is no phylogenetic relation between Veillonellaceae and these thermopilic bacteria; however, recent research on the genome sequences of members of Thermotogales revealed that a large number of genes in Thermotogales show affinities to the Clostridiales (40). Moreover, there are various reports suggesting the relationship between Thermotogales and firmicutes, implying the necessity of re-consideration of the phylogenetic placement of thermophilic ancient bacteria $(41,42)$. These findings imply and support the possibility of the evolutionary relationship between the Veillonellaceae and Thermotogales, despite their having been distantly positioned in a classic phylogenetic tree.

(ii) Phylogenetic distribution of LDC/ODC and its regulatory system

LDC/ODC shows eukaryotic ODC-like properties in respect both of the primary sequence homology and of the enzymatic characteristics, representing the phylogenetically intriguing feature. The homologues of $1 \mathrm{dc} / \mathrm{odc}$ are well distributed among the Veillonellaceae family; thus existence of the eukaryotic ODC may represents the feature of this family. In addition, ldc/odc homologues are also found in Aquifex aeolicus and Tt. maritima, similar to the case in the SLH domain of Mep45. These homologues also possess the deduced antizymebinding region, implying possible occurrence of an antizyme-mediated regulatory system as shown in S. ruminantium LDC/ODC. Indeed, ribosomal protein L10 of these bacteria contains two segments that are demonstrated in P22 to be responsible for binding to LDC/ODC (21). Hence, it seems likely that the antizyme-mediated regulatory process occurs also in these bacteria.

\section{Concluding Remarks}

A series of studies on the "cadaverine-physiology" in $S$. ruminantium have revealed that cadaverine exerts its function in a quite distinctive scheme compared to the general bacteria so far reported. The physiological significance of cadaverine is to mediate the structural linkage between the outer membrane and the peptidoglycan, thereby maintaining the envelope integrity (Fig. 2). Cytoplasmic synthesis of cadaverine works totally in a eukaryotic manner, including antizyme-mediated regulation (Fig. 3). These findings clearly indicate the biological diversity of this bacterium beyond the pre-existing knowledge related to the polyamine-physiology, cell envelope-architecture, and the regulatory system for the enzyme. Further understanding of cadaverine-phys- 
iology in S. ruminantium will lead to expanding the knowledge in these research fields. The major remaining challenges include:

(i) Identification of the gene encoding lipid intermediate:diamine transferase will be the next focus to fully understand the cadaverine-adding reaction into the lipid intermediate of this bacterium.

(ii) Isolation and characterization of the ATP-dependent protease(s) implicated in the antizyme-mediated proteolysis of LDC/ODC. This will enable the complete in vitro reconstitution of the bacterial antizymemediated proteolytic system, or in other words "bacterial proteasome," and lead to further understanding of the molecular mechanism of this system.

In addition to these molecular-level research focuses, it seems worth investigating this organism from the point of view of bacterial phylogeny. S. ruminantium possesses various features contradictory to the phylogenetic classification, such as its Gram-negative cell surface structure, despite its phylogenetic position in Gram-positive bacteria, eukaryotic enzymatic processes for cadaverine synthesis, and unusual relationships to the thermophilic ancient bacteria. There are various reports pointing out the contradiction of $16 \mathrm{~S}$ rRNA-based classification of bacteria, concerning the re-consideration of the phylogenetic tree (41-43). The genome-based characterization of $S$. ruminantium might play a key role for the future consideration of bacterial evolution and phylogeny.

\section{Acknowledgments}

We are grateful to all the people supporting us to recover and continue the research activity from the crisis which occurred on 11th March, 2011. We would like to sincerely express our heartfelt concern for the people still suffering from the disaster, and wish them early and full recovery.

\section{REFERENCES}

1) Salton MRJ. 1994. The bacterial cell envelope-a historical perspective. In: Bacterial Cell Wall (Ghuysen JM, Hakenbeck R, eds), p 1-20. Elsevier, Amsterdam.

2) Bernadac A, Gavioli M, Lazzaroni JC, Raina S, Lloubés R. 1998. Escherichia coli tol-pal mutants form outer membrane vesicles. J Bacteriol 180: 4872-4878.

3) Fung J, MacAlister TJ, Rothfield LI. 1978. Role of murein lipoprotein in morphogenesis of the bacterial division septum: phenotypic similarity of $l k y D$ and lpo. J Bacteriol 133: 1467-1471.

4) Godlewska R, Wiśniewska K, Pietras Z, JagusztynKrynicka EK. 2009. Peptidoglycan-associated lipoprotein (Pal) of Gram-negative bacteria: function, structure, role in pathogenesis and potential application in immunoprophylaxis. FEMS Microbiol Lett 298: 1-11.

5) Suzuki H, Nishimura Y, Yasuda S, Nishimura A, Yamada M, Hirota Y. 1978. Murein-lipoprotein of Escherichia coli: A protein involved in the stabilization of bacterial cell envelope. Mol Gen Genet 167: 1-9.

6) Yem DW, Wu HC. 1978. Physiological characterization of an Escherichia coli mutant altered in the structure of murein lipoprotein. J Bacteriol 133: 1419-1426.

7) Sturgis JN. 2001. Organisation and evolution of the tolpal gene cluster. J Mol Microbiol Biotechnol 3: 113-122.
8) Kamio Y, Takahashi H. 1980. Isolation and characterization of outer and inner membrane of Selenomonas ruminantium lipid compositions. J Bacteriol 141: 888898.

9) Kamio Y, Itoh Y, Terawaki Y. 1981. Chemical structure of peptidoglycan in Selenomonas ruminantium: Cadaverine links covalently to the D-glutamic acid residue of peptidoglycan. J Bacteriol 146: 49-53.

10) Kamio Y, Itoh Y, Terawaki Y, Kusano T. 1981. Cadaverine is covalently linked to peptidoglycan in Selenomonas ruminantium. J Bacteriol 145: 122-128.

11) Kamio Y, Pösö H, Terawaki Y, Paulin L. 1986. Cadaverine covalently linked to a peptidoglycan is an essential constituent of the peptidoglycan necessary for the normal growth in Selenomonas ruminantium. J Biol Chem 261: 6585-6589.

12) Kojima S, Ko KC, Takatsuka $Y$, Abe N, Kaneko J, Itoh $Y$, Kamio Y. 2010. Cadaverine covalently-linked to the peptidoglycan mediates the interaction between the peptidoglycan and periplasm-exposed SLH domain of major outer membrane protein Mep45 in Selenomonas ruminantium. J Bacteriol 192: 5953-5961.

13) Kojima S, Kaneko J, Abe N, Takatsuka Y, Kamio Y. 2011. Cadaverine covalently linked to the peptidoglycan serves as the correct constituent for the anchoring mechanism between the outer membrane and the peptidoglycan in Selenomonas ruminantium. J Bacteriol 193: 2347-2350.

14) Tabor CW, Tabor H. 1985. Polyamines in microorganisms. Microbiol Rev 49: 81-99.

15) Kamio Y, Terawaki Y. 1983. Purification and properties of Selenomonas ruminantium lysine decarboxylase. J Bacteriol 153: 658-664.

16) Takatsuka Y, Onoda M, Sugiyama T, Muramoto K, Tomita T, Kamio Y. 1999. Novel characteristics of Selenomonas ruminantium lysine decarboxylase capable of decarboxylating both L-lysine and L-ornithine. Biosci Biotechnol Biochem 63: 1063-1069.

17) Takatsuka Y, Yamaguchi Y, Ono M, Kamio Y. 2000. Gene cloning and molecular characterization of lysine decarboxylase from Selenomonas ruminantium delineate its evolutionary relationship to ornithine decarboxylase from eukaryotes. J Bacteriol 182: 6732-6741.

18) Kamio Y, Terawaki Y, Izaki K. 1982. Biosynthesis of cadaverine-containing peptidoglycan in Selenomonas ruminantium. J Biol Chem 257: 3326-3333.

19) Yamaguchi Y, Takatsuka Y, Kamio Y. 2002. Identification of a 22-kDa protein required for the degradation of Selenomonas ruminantium lysine decarboxylase by ATPdependent protease. Biosci Biotechnol Biochem 66: 1431-1434.

20) Yamaguchi Y, Takatsuka Y, Matsufuji S, Murakami Y, Kamio Y. 2006. Characterization of a counterpart to mammalian ornithine decarboxylase antizyme in prokaryotes. J Biol Chem 281: 3995-4001.

21) Yamaguchi Y, Takatsuka Y, Kamio Y. 2008. Two segments in bacterial antizyme P22 are essential for binding and enhance degradation of lysine/ornithine decarboxylase in Selenomonas ruminantium. J Bacteriol 190: 442-446.

22) Labishinski H, Maidhof H. 1994. Bacterial peptidoglycan: overview and evolving concepts. In: Bacterial Cell Wall (Ghuysen JM, Hakenbeck R, eds), p 23-35. Elsevier, Amsterdam.

23) Barreteau H, Kovac A, Boniface A, Sova M, Gobec S, Blanot D. 2008. Cytoplasmic steps of peptidoglycan syn- 
thesis. FEMS Microbiol Rev 32: 168-207.

24) Matsuhashi M. 1994. Utilization of lipid-linked precursors and the formation of peptidoglycan in the process of cell growth and division: membrane enzymes involved in the final steps of peptidoglycan synthesis and the mechanism of their regulation. In: Bacterial Cell Wall (Ghuysen JM, Hakenbeck R, eds), p 55-68. Elsevier, Amsterdam.

25) Kamio Y. 1987. Structural specificity of diamines covalently linked to peptidoglycan for cell growth of Veillonella alcalescens and Selenomonas ruminantium. J Bacteriol 169: 4837-4840.

26) Pösö H, McCann PP, Tanskanen R, Bey P, Sjoerdsma A. 1984. Inhibition of growth of Mycoplasma dispar by DL$\alpha$-difluoromethyllysine, a selective irreversible inhibitor of lysine decarboxylase, and reversal by cadaverine (1,5diaminopentane). Biochem Biophys Res Commun 125: 205-210.

27) Sára M, Sleytr UB. 2000. S-Layer proteins. J Bacteriol 182: 859-868.

28) Engelhardt H, Peters J. 1998. Structural research on surface layers: A focus on stability, surface layer homology domains, and surface layer-cell wall interactions. $J$ Struct Biol 124: 276-302.

29) Boeker EA, Snell EE. 1972. Amino acid decarboxylase. Enzymes 6: 217-253.

30) Sabo DL, Boeker EA, Byers B, Waron H, Fisher EH. 1974. Purification and physical properties of inducible Escherichia coli lysine decarboxylase. Biochemistry 13: 662-670.

31) Jäne J, Alhonen-Hongisto L. 1989. Inhibitors of ornithine decarboxylase: Biochemistry and applications. In: Ornithine Decarboxylase: Biology, Enzymology, and Molecular Genetics (Hayashi S, ed), p 59-85. Pergamon Press, New York.

32) Grishin NV, Phillips MA, Goldsmith EJ. 1995. Modeling of the spatial structure of eukaryotic ornithine decarboxylase. Protein Sci 4: 1291-1304.

33) Kern AD, Oliveira MA, Coffino P, Hackert ML. 1999. Structure of mammalian ornithine decarboxylase at 1.6 Å resolution: stereochemical implications of PLP-dependent amino acid decarboxylases. Structure 7: 567-581.

34) Takatsuka Y, Tomita T, Kamio Y. 1999. Identification of the amino acid residues conferring substrate specificity upon Selenomonas ruminantium lysine decarboxylase. Biosci Biotechnol Biochem 63: 1843-1846.

35) Hayashi S, Murakami Y, Matsufuji S. 1996. Ornithine decarboxylase antizyme: a novel type of regulatory protein. Trends Biochem Sci 21: 27-30.

36) Kamio Y, Nakamura K. 1987. Putrescine and cadaverine are constituents of peptidoglycan in Veillonalla alcalescens and Veillonella parvula. J Bacteriol 169: 28812884.

37) Hirao T, Sato M, Shirahata A, Kamio Y. 2000. Covalent linkage of polyamines to peptidoglycan in Anaerovibrio lipolytica. J Bacteriol 182: 1154-1157.

38) Hamana K. 1999. Distribution of cell wall-linked polyamines within the Gram-negative anaerobes of the subbranch Sporomusa belonging phylogenetically to Gram-positive taxa. Microbios 100: 145-157.

39) Hamana K, Saito T, Okada M, Sakamoto A, Hosoya R. 2002. Covalently linked polyamines in the cell wall peptidoglycan of Selenomonas ruminantium, Anaeromusa, Dendrosporobacter, Acidaminococcus and Anaerovibrio belonging to the Sporomusa subbranch. J Gen Appl Microbiol 48: 177-180.

40) Zhaxybayeva O, Swithers KS, Lapierre P, Fournier GP, Bickhart DM, DeBoy RT, Nelson KE, Nesbø CL, Doolittle WF, Gogarten JP, Noll KM. 2009. On the chimeric nature, thermophilic origin, and phylogenetic placement of the Thermotogales. Proc Natl Acad Sci USA 106: 5865-5870.

41) Gupta RS. 1998. Protein phylogenies and signature sequences: a reappraisal of evolutionary relationships among archaeabacteria, eubacteri, and eukayotes. Microbiol Mol Biol Rev 62: 1435-1491.

42) Gupta RS. 2000. The natural evolutionary relationships among prokaryotes. Crit Rev Microbiol 26: 111-131.

43) Nelson KE, Clayton RA, Gill SR, Gwinn ML, Dodson RJ, Haft DH, Hickey EK, Peterson JD, Nelson WC, Ketchum KA, McDonald L, Utterback TR, Malek JA, Linher KD, Garrett MM, Stewart AM, Cotton MD, Pratt MS, Phillips CA, Richardson D, Heidelberg J, Sutton GG, Fleischmann RD, White O, Salzberg SL, Smith HO, Venter JC, Fraser CM. 1999. Evidence for lateral gene transfer between archaea and bacteria from genome sequence of Thermotoga maritima. Nature 399: 323-329. 